\title{
WEAK AMENABILITY OF GROUP ALGEBRAS OF CONNECTED COMPLEX SEMISIMPLE LIE GROUPS
}

\author{
B.E. JOHNSON
}

(Communicated by Palle E. T. Jorgensen)

\begin{abstract}
We consider the problem of whether every continuous derivation from a group algebra $L^{1}(G)$ into its dual $L^{\infty}(G)$ (where the $L^{1}(G)$ actions on $L^{\infty}(G)$ are the adjoint of multiplication in $L^{1}(G)$ ) is inner, that is, of the form $D(a)=a F-F a$ for some $F \in L^{\infty}(G)$. This had previously been established to hold for discrete and amenable groups and is now established for $G=\mathrm{Gl}(n, \mathbf{C})$ and for all connected semisimple complex Lie groups.
\end{abstract}

\section{INTRODUCTION}

The dual $A^{*}$ of a Banach algebra $A$ is an $A$-bimodule under the operations

$$
\begin{aligned}
& (a F, b)=(F, b a), \\
& (F a, b)=(F, a b), \quad a, b \in \mathbf{A}, F \in \mathbf{A}^{*} .
\end{aligned}
$$

The algebra $\mathbf{A}$ is weakly amenable if every continuous derivation from $\mathbf{A}$ into $A^{*}$ is inner, that is, of the form $a \mapsto a F-F a$ for some $F \in \mathbf{A}^{*}$. In [3] the author showed that, for a discrete group, and more generally for a SIN group (that is a group for which the group algebra has a central approximate identity), the group algebra $L^{1}(G)$ is weakly amenable. In that paper the fact that the group algebra of the matrix group $\operatorname{Gl}(n, \mathbf{C})$ is weakly amenable was announced without proof. In this paper we prove the result for $\mathrm{Gl}(n, \mathbf{C})$ (Corollary 2.4) and extend the method to show that the group algebra of any connected semisimple complex Lie group is weakly amenable. Before going into the details it is worthwhile to indicate why the result is rather easy for discrete groups, how the extensions we have made are possible and why the case of a general locally compact group has not been settled. By the well known correspondence between continuous linear maps from $\mathbf{A}$ into $\mathbf{A}^{*}$ and elements of $(\mathbf{A} \hat{\otimes} \mathbf{A})^{*}$ and the identity $L^{1}(G) \hat{\otimes} L^{1}(G) \simeq L^{1}(G \times G)$, a derivation $D$ from $\mathbf{A}$ into $\mathbf{A}^{*}$ corresponds via the identity

$$
(D a, b)=\iint F(g, h) a(g) b(h) d g d h
$$

Received by the editors November 7, 1989.

1980 Mathematics Subject Classification (1985 Revision). Primary 43A20. 
to an element $F$ of $L^{\infty}(G \times G)$ with

$$
F(g h, k)=F(h, k g)+F(g, h k),
$$

for almost all $(g, h, k) \in G \times G \times G$. Our method depends on being able to say that there is a bounded measurable function $F^{\prime}$ on $G \times G$ which is equal to $F$ almost everywhere for which the relationship * holds for all $g, h, k \in G$. Techniques exist for this but they all involve some degree of commutativity between the approximate identities used and the group operations. For the case of $G=\mathrm{Gl}(n, \mathrm{C})$ the crucial feature is that, for the action of $G$ on $G$ by conjugation, a $G$ invariant subset, namely, the set of matrices with distinct eigenvalues, has complement of measure zero and is the product of a $G$ space $X$ on which $G$ acts trivially and the $G$ space $G / H$ of right cosets of the subgroup $H$ of diagonal matrices (and $G$ acts by right translation). For any bounded measure $\mu$ on $X$, integration of functions on this product space with respect to $\mu$ gives a map from $L^{\infty}(G)$ onto $L^{\infty}(G / H)$ which commutes with the $G$ action.

The body of the paper is divided into two sections, one dealing with our general method and obtaining the result for $\mathrm{Gl}(n, \mathbf{C})$ and the other with general semisimple Lie groups.

\section{THE GENERAL METHOD}

Theorem 2.1. Let $G$ be a locally compact group with a $D^{\prime}$ sequence $\left\{U_{n}\right\}$ (see $\left[1\right.$, p. 637]), and let $\mathbf{X}$ be a Banach space. On $L^{1}(G) \hat{\otimes} \mathbf{X}$ define the structure of an $L^{1}(G)$-bimodule by

$$
\begin{aligned}
& b(a \otimes x)=\lambda(b) a \otimes x, \\
& (a \otimes x) b=a b \otimes x, \quad a, b \in L^{1}(G), x \in \mathbf{X},
\end{aligned}
$$

where $\lambda$ is a left Haar measure on $G$. Then every continuous derivation from $L^{1}(G)$ into $\left(L^{1}(G) \hat{\otimes} \mathbf{X}\right)^{*}$ is inner.

Proof. Let $D$ be a continuous derivation from $L^{1}(G)$ into $\left(L^{1}(G) \hat{\otimes} \mathbf{X}\right)^{*}$. Then there is a trilinear functional $S$ with $S(a, b, x)=(D(a), b \otimes x)\left(a, b \in L^{1}(G)\right.$, $x \in \mathbf{X})$. Let LIM denote a Banach limit on $\mathbb{N}$ and define

$$
\widetilde{S}(g, h, x)=\operatorname{LIM}_{m} \operatorname{LIM}_{n} S\left(\delta_{g} e_{m}, \delta_{h} e_{n}, x\right), \quad g, h \in G, x \in \mathbf{X},
$$

where $e_{m}=\lambda\left(U_{m}\right)^{-1} \times$ characteristic function of $U_{m}$ and $\delta_{g}$ is unit mass situated at $g . \tilde{S}$ is linear in $x$ and $|\widetilde{S}(g, h, x)| \leq\|D\|\|x\|$.

The derivation law for $D$ gives

$$
\begin{aligned}
S\left(\delta_{g} e_{m} \delta_{h} e_{n}, \delta_{k} e_{p}, x\right)=S\left(\delta_{g} e_{m}, \delta_{k} e_{p}, x\right)+S\left(\delta_{h} e_{n}, \delta_{k} e_{p} \delta_{g} e_{m}, x\right), \\
g, h, k \in G, \quad m, n, p \in \mathbb{N},
\end{aligned}
$$

because $\lambda\left(\delta_{h} e_{n}\right)=1$. We now apply LIM to each side of this equation, first on $p$, then on $m$ and finally on $n$. Because $e_{m} \delta_{h} e_{n} \rightarrow \delta_{h} e_{n}$ in $L^{1}(G)$ norm 
as $m \rightarrow \infty$, we get

$$
\tilde{S}(g h, k, x)=\tilde{S}(g, k, x)+\tilde{S}(h, k g, x), \quad g, h, k \in G, x \in \mathbf{X} .
$$

Now put $\tilde{\alpha}(g, x)=\widetilde{S}(g, e, x), g \in G, x \in \mathbf{X}$. We have $\tilde{S}(g, k, x)=$ $\widetilde{S}(g, e k, x)=\tilde{\alpha}(k g, x)-\tilde{\alpha}(k, x)$. To complete the proof we have to show that, for each $x \in \mathbf{X}$, the functions $g, h \mapsto \tilde{S}(g, h, x)$ and $g \mapsto \tilde{\alpha}(g, x)$ are bounded measurable functions, and so we can define $\alpha \in\left(L^{1}(G) \hat{\otimes} X\right)^{*}$ by

$$
\alpha(a \otimes x)=\int a(g) \tilde{\alpha}(g, x) d g .
$$

This would give

$$
\begin{aligned}
(a \alpha-\alpha a, b, x) & =\iint(\tilde{\alpha}(h g, x)-\tilde{\alpha}(h, x)) a(g) b(h) d g d h \\
& =\iint \tilde{S}(g, h, x) a(g) b(h) d g d h,
\end{aligned}
$$

where the last integral has to be shown to be $S(a, b, x)=(D(a), b, x)$. For each $x \in \mathbf{X}$, there is $\sigma_{x} \in L^{\infty}(G \times G)$ with

$$
S(a, b, x)=\iint \sigma_{x}(g, h) a(g) b(h) d g d h .
$$

For each value of $m$ and $g, h \rightarrow \int \sigma_{x}(r, h)\left(\delta_{g} e_{m}\right)(r) d r$ is an $L^{\infty}(G)$ function, and so, by [1, Theorem 44.18], $\lim _{n} \iint \sigma_{x}(r, s)\left(\delta_{g} e_{m}\right)(r)\left(\delta_{h} e_{n}\right)(s) d r d s$ exists and equals $\int \sigma_{x}(r, h)\left(\delta_{g} e_{m}\right)(r) d r$ for almost all $h \in G$. Amalgamating the exceptional sets for the different values of $m$ we see that, for each $x$, there is a subset $E_{1}$ of $G \times G$ such that

$$
\lim _{n} S\left(\delta_{g} e_{m}, \delta_{h} e_{n}, x\right)=\int \sigma_{x}(r, h)\left(\delta_{g} e_{m}\right)(r) d r
$$

for all $m$ and all $(g, h) \in G \times G \backslash E_{1}$ and, for each $g,\left\{h ;(g, h) \in E_{1}\right\}$ is of measure zero. However, $(g, h) \mapsto S\left(\delta_{g} e_{m}, \delta_{h} e_{n}, x\right)$ is a continuous function, so the set of points on which this limit exists is measurable. Thus the expression on the right is a measurable function and $E_{1}$ is a measurable set, hence a set of measure zero. A similar argument now shows that, for each $x$,

$$
\lim _{m} \int \sigma_{x}(r, h)\left(\delta_{g} e_{m}\right)(r) d r=\sigma_{x}(g, h)
$$

except for a set of values of $(g, h)$ of measure zero. Thus

$$
\sigma_{x}(g, h)=\lim _{m} \lim _{n} S\left(\delta_{g} e_{m}, \delta_{h} e_{n}, x\right)=\widetilde{S}(g, h, x)
$$

except for a set of values of $(g, h)$ of measure zero. This shows that $\widetilde{S}$ is a measurable function of $g$ and $h$ and that

$$
S(a, b, x)=\iint \widetilde{S}(g, h, x) a(g) b(h) d g d h .
$$


The function $g \mapsto \widetilde{S}(g, h, x)$ is thus measurable for one value $h_{0}$ (in fact for almost all values) of $h$. The identity

$$
\widetilde{S}(g, h, x)=\widetilde{S}\left(g, h_{0} h_{0}^{-1} h, x\right)=\widetilde{S}\left(h_{0}^{-1} h g, h_{0}, x\right)-\widetilde{S}\left(h_{0}^{-1} h, h_{0}, x\right)
$$

then shows that it is measurable for all values of $h$ and hence that $\tilde{\alpha}(g, x)$ is a measurable function of $g$ for each $x \in \mathbf{X}$.

Corollary 2.2. Under the same conditions as in Theorem 2.1 , let $H$ be a closed subgroup of $G$. The space $L^{1}(G / H)$ of bounded measures on the right coset space $G / H$, which are absolutely continuous with respect to the quotient measure space structure on $G / H$ coming from the Haar measure space structure on $G$, is a quotient bimodule of the bimodule $L^{1}(G)$, and every continuous derivation from $L^{1}(G)$ into $\left(L^{1}(G / H) \hat{\otimes} \mathbf{X}\right)^{*}$ is inner.

Proof. Let $q$ be the quotient map of $L^{1}(G)$ onto $L^{1}(G / H)$ and $D_{0}$ be a continuous derivation from $L^{1}(G)$ into $\left(L^{1}(G / H) \hat{\otimes} \mathbf{X}\right)^{*}$. Then $q_{0}=\left(q \otimes \operatorname{Id}_{\mathbf{X}}\right)^{*}$ is a bimodule map from $\left(L^{1}(G / H) \hat{\otimes} \mathbf{X}\right)^{*}$ into $\left(L^{1}(G) \hat{\otimes} \mathbf{X}\right)^{*}$, so $D=q_{0} D_{0}$ is a derivation and hence inner, by the theorem. Applying the proof of the theorem to the present case, we have $S(a, b, x)=\left(D_{0}(a), q b, x\right)$. For any $h$ in $H$, $q(b)=q\left(\delta_{h} b\right)$ and so $\tilde{S}(g, h k, x)=\tilde{S}(g, k, x)$ for all $g, k \in G, h \in H$ and $x \in \mathbf{X}$. We thus have

$$
\tilde{S}(h g, e, x)=\tilde{S}(h, e, x)+\widetilde{S}(g, h, x)=\tilde{S}(h, e, x)+\widetilde{S}(g, e, x)
$$

for $g \in G, h \in H$ and, putting $g=h^{n}$, we see by induction that $\widetilde{S}\left(h^{n}, e, x\right)=$ $n \widetilde{S}(h, e, x)$, implying $\widetilde{S}(h, e, x)=0$ because $\widetilde{S}$ is bounded. Hence $\tilde{\alpha}(h g, x)$ $=\widetilde{S}(h g, e, x)=\widetilde{S}(g, e, x)$, that is, $g \mapsto \tilde{\alpha}(g, x)$ is constant on the right cosets of $H$ and can be considered as an element of $L^{\infty}(G / H)$. Thus defining $\alpha_{0}\left(a_{0}, x\right)=\int \tilde{\alpha}(H g, x) d a_{0}(H g)$, we get $\alpha_{0} \in\left(L^{1}(G / H) \hat{\otimes} \mathbf{X}\right)^{*}$ and $\alpha_{0}(q(a), x)$ $=\alpha(a, x)$. Thus $D(a)=a \alpha-\lambda(a) \alpha$ implies $D_{0}(a)=a \alpha_{0}-\lambda(a) \alpha_{0}$.

Lemma 2.3. Let $G$ be a real Lie group. Then $G$ contains a $D^{\prime}$ sequence.

Proof. Suppose $G$ is $d$ dimensional. We take the Euclidean metric on $\mathbf{R}^{d}$ and a cononical chart $\alpha$ mapping a neighbourhood $U$ of $e$ in $G$ onto an open ball $B_{\varepsilon}$ of radius $\varepsilon$ centre at 0 in $\mathbf{R}^{d}$. There is a neighbourhood $V$ of $e$ such that $V V^{-1} \subseteq U$ and $\alpha$ maps $V$ onto $B_{\eta}$ for some $\eta<\varepsilon$. The map $\pi: B_{\eta} \times B_{\eta} \rightarrow B_{\varepsilon}$ given by $\pi(x, y)=\alpha\left(\left(\alpha^{-1} x\right)\left(\alpha^{-1} y\right)^{-1}\right)$ is a smooth map with $\|\pi(x, y)-(x-y)\| \leq K\left(\|x\|^{2}+\|y\|^{2}\right)$ for all $x, y \in B_{\eta}$ for some $K$. Thus, for $r<\min \left\{\eta,(2 K)^{-1}\right\}$, if $x, y \in B_{r}$ then $\|\pi(x, y)\| \leq\|x\|+\|y\|+2 K r^{2} \leq 3 r$, that is $\pi\left(B_{r}, B_{r}\right) \subseteq B_{3 r}$.

Under the mapping $\alpha$, left Haar measure $\lambda$ on $U$ is carried into a measure on $B_{\varepsilon}$ which is absolutely continuous with respect to Lebesgue measure $L$, and we have, for a suitably chosen Haar measure,

$$
\int \phi(\alpha(g)) d \lambda(g)=\int \phi(x) J(x)^{-1} d x
$$


for all $\phi \in C_{0}\left(B_{\varepsilon}\right)$ where $J(x)=\operatorname{det}\left[\partial \pi_{i}^{\prime}(x, y) / \partial y_{j}\right]_{y=0}$ and $\pi^{\prime}(x, y)=$ $\alpha\left(\left(\alpha^{-1} x\right)\left(\alpha^{-1} y\right)\right)$. Thus $J$ is continuous and $J(0)=1$; so, for sufficiently small values of $r$, we have $L\left(B_{r}\right) / 2 \leq \lambda\left(\alpha^{-1} B_{r}\right) \leq 2 L\left(B_{r}\right)$. This shows that $\lambda\left(\left(\alpha^{-1} B_{r}\right)\left(\alpha^{-1} B_{r}\right)^{-1}\right) \leq 2 L\left(\pi\left(B_{r}, B_{r}\right)\right) \leq 2 L\left(B_{3 r}\right) \leq 2.3^{d} L\left(B_{r}\right) \leq 4.3^{d} \lambda\left(\alpha^{-1} B_{r}\right)$ and we can construct a $D^{\prime}$ sequence by taking $U_{n}=\alpha^{-1}\left(B_{r_{n}}\right)$ for a suitable null sequence of values of $r$.

Corollary 2.4. $L^{1}(\mathrm{Gl}(n, \mathrm{C}))$ is weakly amenable.

Proof. Starting with $\mathbf{X}=L^{1}(G)$ considered as an $L^{1}(G)$-bimodule, where the left and right actions are algebra multiplication, and carrying out the transformations in [3, p.31], we get a new $L^{1}(G)$-bimodule structure on $L^{1}(G)$ given by

$$
\begin{aligned}
& (a \circ f)(s)=\lambda(a) f(s) \\
& (f \circ a)(s)=\int f\left(g s g^{-1}\right) a(g) \Delta\left(g^{-1}\right) d g, \quad a \in L^{1}(G), f \in L^{1}(G), s \in G
\end{aligned}
$$

We will have proved the theorem if we show that every continuous derivation from $L^{1}(G)$ into $\left(L^{1}(G)^{\circ}\right)^{*}$ is inner.

The new module action on $L^{\infty}(G)$ is

$$
\begin{aligned}
F \circ a & =\lambda(a) F \\
(a \circ F)(s) & =\int a(g) F\left(g^{-1} s g\right) d g, \quad a \in L^{1}(G), F \in L^{\infty}(G), s \in G .
\end{aligned}
$$

Let $H$ be the subgroup of $G=\operatorname{Gl}(n, \mathbb{C})$ consisting of diagonal matrices, and consider the map $\gamma$ of $G / H \times H$ onto $G$ given by $(H g, h) \mapsto g^{-1} h g$ (this map is well defined because $H$ is abelian). If we restrict this map to a map $G / H \times$ $H_{0} \rightarrow G_{0}$, where $G_{0}, H_{0}$ are the subsets of $G, H$ respectively of elements with $n$ distinct eigenvalues, we have an $n$ !-fold covering map; replacing $H_{0}$ by $X$, the set of matrices $\operatorname{diag}\left(a_{1}, a_{2}, \ldots, a_{n}\right)$, where $\operatorname{Re} a_{i}<\operatorname{Re} a_{j}$ or $\operatorname{Re} a_{i}=\operatorname{Re} a_{j}$ and $\operatorname{Im} a_{i}<\operatorname{Im} a_{j}$ whenever $i<j$, we get a bijective map $\gamma_{0}$ of $G / H \times X$ onto $G_{0}$. If we take the induced Haar measure space structure on $G / H$ and $G_{0}$ and Lebesgue measure on $X, \gamma_{0}$ is a homomorphism and $G \backslash G_{0}$ is of measure zero. Thus $\gamma_{0}$ induces an isometric isomorphism $\gamma$ of $L^{1}(G / H) \widehat{\otimes}\left(L^{1}(X)\right)$ onto $L^{1}(G)$ by

$$
[\gamma(a \otimes x)](k)=a(H g) x(\xi),
$$

where $k \in G_{0}, \xi \in X$ and $g \in G$ with $g^{-1} \xi g=k$. Because

$$
\gamma[(a \otimes x) b](k)=\int a(H g) b\left(g^{-1} r\right) x(\xi) d g,
$$

where $r^{-1} \xi r=k$, and

$$
\begin{aligned}
{[\gamma(a \otimes x) \circ b](k) } & =\int(\gamma a \otimes x)\left(t k t^{-1}\right) b(t) \Delta\left(t^{-1}\right) d t \\
& =\int a(H s) x(\eta) b(t) \Delta\left(t^{-1}\right) d t
\end{aligned}
$$


where $s^{-1} \eta s=t k t^{-1}$ so that $\eta=\xi$ and $t=s^{-1} r$, we see that $\gamma[(a \otimes x) \circ b]=$ $\gamma(a \otimes x) \circ b, a \in L^{1}(G / H), b \in L^{1}(G), x \in L^{1}(X)$. Trivially the left module action is also preserved by $\gamma$, and thi result follows from 2.2 and 2.3 .

\section{THE MAIN THEOREM}

Theorem 3.1. Let $G$ be a connected complex semisimple Lie group. Then $L^{1}(G)$ is weakly amenable.

Proof. We consider open sets $R$ in $G$ for which there is a homeomorphism and measure space isomorphism $\gamma$ from $G / H \times X$ onto $R$, where $H$ is some closed subgroup of $G$ and $X$ is an open set in a Euclidean space with Haar/Lebesgue measure structure such that $\gamma(H g k, \xi)=k^{-1} \gamma(H g, \xi) k, g, k \in G, \xi \in X$. As in the proof of Corollary 2.4, we need to prove that every continuous derivation $D$ from $L^{1}(G)$ into $\left(L^{1}(G)^{\circ}\right)^{*}=L^{\infty}(G)$ is inner. By restriction of $L^{\infty}(G)$ functions to $R, D$ gives a derivation from $L^{1}(G)$ into $L^{\infty}(R)$ which is isomorphic as an $L^{1}(G)$ bimodule with $L^{\infty}(G / H \times X)=\left(L^{1}(G / H) \hat{\otimes} L^{1}(X)\right)^{*}$. Using 2.2 and 2.3 we see that there is an $F \in L^{\infty}(R)$, with $D(a)=a \circ F-F \circ a$ on $R$ for all $a \in L^{1}(G)$ and $\|F\| \leq\|D\|$. The main part of the proof will be to establish that the union of all such sets $R$ has complement in $G$ of measure zero. Because the topology of $G$ has a countable base, this would imply that there is a sequence $\left\{R_{i}\right\}$ of such sets whose union has complement of measure zero. If we take the elements $F_{i}$ of $L^{\infty}\left(R_{i}\right)$ given by the above and define $F(g)=F_{i}(g)$, for $g \in R_{i} \backslash \cup_{j<i} R_{j}$, then $F \in L^{\infty}(G)$ and $D(a)=a \circ F-F \circ a, a \in L^{1}(G)$, completing the proof.

Let $L$ be the Lie algebra of $G$. First of all we consider the case in which the adjoint representation is faithful and $G$ is a closed subgroup of $\mathrm{Gl}(L)$. Taking a basis in $L, G$ is a closed subgroup of $\operatorname{Gl}(n, \mathbf{C})$ where $n=\operatorname{dim} L$. We shall need the following well-known result.

Lemma 3.2. Let $f$ be an analytic function on the open polydisc $D_{1} \times D_{2} \times \cdots \times D_{d}$ in $\mathbf{C}^{d}$. Then, either $f$ is identically zero or its zero set is of $2 d$ Lebesgue measure zero.

Proof. We prove this by induction on $d$. The case $d=1$ is an elementary result. If it holds for $d-1$ and $f$ is analytic on $D_{1} \times \cdots \times D_{d}$ and not identically zero, then consider the functions $\left(z_{2}, \ldots, z_{d}\right) \mapsto f\left(z_{1}, z_{2}, \ldots, z_{d}\right)$. Suppose the set of $z_{1} \in D_{1}$, for which this is identically zero, has a point of accumulation in $D_{1}$. Then, for each $\left(z_{2}, \ldots, z_{d}\right) \in D_{2} \times \cdots \times D_{d}$, the function $z_{1} \mapsto$ $f\left(z_{1}, z_{2}, \ldots, z_{d}\right)$ is identically zero, that is, $f$ is identically zero. Thus, for all but a countable set of values of $z_{1}$, the zero set of $f$ intersects $\left\{z_{1}\right\} \times D_{2} \times$ $D_{3} \times \cdots \times D_{d}$ in a set of $2(d-1)$ measure zero, and the proof is completed by Fubini's Theorem.

An element $g$ of $G$ is called regular if the dimension of the null space of $g-I$ is the rank of $L$. We denote the set of regular elements of $G$ by $G_{1}$. Let 
$p \in\{1, \ldots, n\}$, and let $m(g)$ be the determinant of a fixed $p \times p$ minor of $g-I$. Then, either $m(g) \equiv 0$ or the zero set of $m$ is a closed nowhere dense set of zero measure. If $p>n-r$, then dim range ad $\ell \leq n-r<p$ for all $\ell \in L$, so, for all $\ell$ in a neighbourhood of 0 in $L$, dim $\operatorname{range}(\exp a d \ell-I)<p$ and $m(\operatorname{expad} \ell)=0$, hence $m \equiv 0$. Thus dim range $(g-I) \leq n-r$ for all $g \in G$. If $g \in G_{1}$ then there is an $(n-r) \times(n-r)$ minor $m$, with $m(g) \neq 0$ and $m\left(g^{\prime}\right) \neq 0$ for all $g^{\prime}$ near $g$, that is dim range $\left(g^{\prime}-I\right) \geq n-r$. Then dim range $\left(g^{\prime}-I\right)=n-r$, and hence $G_{1}$ is open. Its complement is the set of common zeros of all the $(n-r) \times(n-r)$ minors and is of measure zero, because the only alternative is that all these minors are identically zero and, if $\ell$ is a regular element of $L$ then, for small positive $t, \operatorname{dim} \operatorname{range}(\exp \operatorname{ad} t \ell)-I=$ dim range $\ell=n-r$ so that one of the $(n-r) \times(n-r)$ minors of $(\operatorname{expad} t \ell)-I$ is not zero.

By the elementary theory of equations, if $P \in \mathbf{C}[x]$ then there is a $Q \in \mathbf{C}[x]$ such that the zeros of $Q$ are $\left(z_{i}-z_{j}\right)^{2}, 1 \leq i<j \leq \operatorname{deg} P$ where the $z_{i}$ are the zeros of $P$. The coefficients of $Q$ are polynomials in the coefficients of $P$. If $P$ is the characteristic function of $g \in G$ then $Q$ is of the form $a_{0}(g)+a_{1}(g) x+a_{2}(g) x^{2}+\ldots$, where the $a_{i}(g)$ are analytic functions on $G$. Thus

$$
F_{k}=\{g: 0 \text { is a zero of } Q \text { of multiplicity } \geq k\}
$$

is either $G$ or a closed nowhere dense subset of $G$ of measure zero. As $\operatorname{dim} \operatorname{ker}(g-I) \geq r$ for all $g \in G, F_{k_{0}}=G$ for $k_{0}=r(r-1) / 2$. We shall show that $F_{k_{0}+1} \neq G$. Suppose $C$ is a Cartan subalgebra of $L$ and $\mathscr{R}$ is the set of roots of $C$ (including the zero root). Put

$$
E=\{c ; c \in C, r(c) \neq s(c) \text { whenever } r, s \in \mathscr{R} \text { and } r \neq s\} .
$$

Since $E$ is the complement of a finite set of hyperplanes, it is a dense open subset of $C$. If $c \in E$ then 0 is an eigenvalue of ad $c$ with multiplicity $r$ and the eigenvalues are otherwise distinct. Thus, for sufficiently small $c \in E, 1$ is an eigenvalue of $\exp \operatorname{ad} c$ and the eigenvalues are otherwise distinct, so expad $c \notin$ $F_{k_{0}+1}$. We put $G_{0}=G_{1} \cap\left(G \backslash F_{k_{0}+1}\right)$ so that $G_{0}$ is an open dense subset of $G$ with $G \backslash G_{0}$ of measure zero. We have $g \in G_{0}$ if and only if there is a basis for $L$ with respect to which $g$ has a diagonal matrix with 1 in the first $r$ places and $n-r$ distinct elements of $\mathbf{C} \backslash\{0,1\}$ on the rest of the diagonal. We denote the set of diagonal matrices of this form by $\Delta_{r}$.

Let $g \in G_{0}$ and choose a basis of $L$ such that $g \in \Delta_{r}$. Then the set $Z_{g}=\{h, h \in G, g h=h g\}$ is a closed subgroup of $G$ with Lie algebra

$$
\begin{aligned}
K & =\{\ell: \ell \in L, \exp (t \operatorname{ad} \ell) g=g \exp (t \operatorname{ad} \ell, t \in \mathscr{R}\} \\
& =\{\ell: \ell \in L, \operatorname{ad} \ell g=g \operatorname{ad} \ell\} \\
& =\{\ell: \ell \in L, g \ell=\ell\},
\end{aligned}
$$


that is, $K$ is the linear span of the first $r$ basis vectors. Let $V$ be a neighbourhood of 0 in $K$ such that, for all $k \in V$, all matrix entries in ad $k$ have modulus less that $\delta=\left(\inf \log \left|\alpha \beta^{-1}\right|\right) / 2$, where the infimum is taken over all pairs of distinct eigenvalues $\alpha, \beta$ of $g$. Because $g \in G_{0}$, the matrix of ad $k$ for any $k \in K$ has nonzero off-diagonal entries $k_{i j}$ only for $i, j \leq r$. However, if $k \in K$, then, choosing $t \in \mathbf{R}$ with $t k \in V, g$ expad $t k$ also has this form and has distinct entries different from 1 on the diagonal outside the first $r$ rows. Then, since $\operatorname{dim} \operatorname{ker}(g \exp$ ad $t k-I) \geq r$, we have expad $t k=I$ on $K$, for all such $t$, showing that ad $k$ has a diagonal matrix with zeros in the first $r$ rows.

Replacing $V$ by a smaller neighbourhood of 0 in $K$ if necessary, the exponential map maps $V$ homeomorphically onto a neighbourhood of $I$ in $Z_{g}$. We now consider the map $\gamma: G / Z_{g} \times V \rightarrow G$ given by $\gamma\left(Z_{g} g^{\prime}, v\right)=$ $\left(g^{\prime}\right)^{-1} \exp v g^{\prime}$. Because $g \exp v \in Z_{g}$, this map depends only on the coset $Z_{g} g^{\prime}$ and is a well defined continuous map of $G / Z_{g} \times V$ into $G$ with $\gamma\left(Z_{g} g^{\prime} k, v\right)=$ $k^{-1} \gamma\left(Z_{g} g^{\prime}, v\right) k$. We now show that it is injective. If $\gamma\left(Z_{g} k, v\right)=\gamma\left(Z_{g} k^{\prime}, v^{\prime}\right)$ then $k\left(k^{\prime}\right)^{-1} g \exp v^{\prime}=g \exp v k\left(k^{\prime}\right)^{-1}$, where $g$, ad $v$ and ad $v^{\prime}$ are given by diagonal matrices $g=\operatorname{diag}\left(g_{1}, \ldots, g_{n}\right)$, ad $v=\operatorname{diag}\left(v_{1}, \ldots, v_{n}\right)$ and $\operatorname{ad} v^{\prime}=\operatorname{diag}\left(v_{1}^{\prime}, \ldots, v_{n}^{\prime}\right)$. If $k\left(k^{\prime}\right)^{-1}$ is given by $\left[\kappa_{i j}\right]$, then this equation is

$$
\kappa_{i j} g_{j} \exp v_{j}^{\prime}=g_{i} \exp v_{i} \kappa_{i j} \quad i, j=1, \ldots, n .
$$

By the definition of $V,\left|v_{i}\right|<\delta$ and $\left|v_{i}^{\prime}\right|<\delta$, so $g_{j} \exp v_{j}^{\prime}=g_{i} \exp v_{i}$ if and only if $i \leq r$ and $j \leq r$ or $i=j$ and $v_{i}=v_{j}^{\prime}$. Thus $\left[\kappa_{i j}\right]$ has nonzero offdiagonal entries only in the principal $r \times r$ minor, which implies that $k\left(k^{\prime}\right)^{-1}$ commutes with $g, \exp v$ and $\exp v^{\prime}$. Thus $Z_{g} k=Z_{g} k^{\prime}, \exp v=\exp v^{\prime}$ and hence $v=v^{\prime}$. Finally we show that the range of $\gamma$ is open and $\gamma$ is a measure space isomorphism by showing its derivative is nonsingular everywhere. Since the map $k^{\prime} \mapsto k^{-1} k^{\prime} k$ is an analytic homeomorphism on $G$, it is enough to show that $\gamma^{\prime}$ is nonsingular at the points $\left(Z_{g}, v\right), v \in V$. Let $v_{0} \in V$ and $M$ be the subspace of $L$ spanned by the eigenvectors $e_{r+1}, \ldots, e_{n}$ of $g$ not in $K$. The maps $\ell \mapsto g \exp v_{0} \exp \ell, k \mapsto v_{0}+k, m \mapsto Z_{g} \exp m$ are diffeomorphisms of neighbourhoods $L_{0}, K_{0}$ and $M_{0}$ of 0 in $L, K$ and $M$ onto neighbourhoods of $g \exp v_{0}$ in $G$, of $v_{0}$ in $K$ and of $Z_{g}$ in $G / Z_{g}$, and so $\gamma$ gives rise to a diffeomorphism $\Gamma$ of $M_{0} \times K_{0}$ into $L_{0}$. We have

$$
\exp (-m) g \exp \left(v_{0}+k\right) \exp m=g \exp v_{0} \exp \Gamma(m, k) .
$$

Thus the differential $\Gamma_{0}^{\prime}$ of $\Gamma$ at $m=0, k=0$ is given by $\Gamma_{0}^{\prime}\left(m^{\prime}, k^{\prime}\right)=$ $\left(g \exp v_{0}-I\right) m^{\prime}+k^{\prime}$ so that $\Gamma_{0}^{\prime}$ is given by a diagonal matrix with 1 in the first $r$ places and nonzero entries everywhere else on the diagonal. We have thus shown that there is a set $R$ in $G$ with the properties described at the beginning of this proof with $g \in R$, and the result follows for $G$. 
We now turn to the case in which $G$ has a possibly nontrivial centre $Z$, a finite subgroup. Let $q$ denote the quotient map $G \rightarrow G / Z$. We apply the above analysis to $G / Z$ and put $G_{0}=q^{-1}(G / Z)_{0}$. For any $g \in G$ we have $Z_{g} \subseteq q^{-1} Z_{q g}$, and if $h \in q^{-1} Z_{q g}$ then $q\left(g h g^{-1} h^{-1}\right)=e$, that is, $g h g^{-1} h^{-1} \in Z$ so that if $h$ is close to $e$ then $h \in Z_{g}$. Thus $Z_{g}$ is an open subgroup of $q^{-1} Z_{q g}$, and $Z_{g}$ and $Z_{q g}$ have the same Lie algebra. Also $G$ and $G / Z$ have the same Lie algebra. Take $g \in G_{0}$. The construction of $V$ and $\gamma$ is as before $(\alpha, \beta$ in the definition of $V$ refer to the eigenvalues of ad $g$ ). In showing that $\gamma$ is injective, we start with $\gamma\left(Z_{g} k, v\right)=\gamma\left(Z_{g} k^{\prime}, v^{\prime}\right)$ and work as above in $G / Z$ to see that $v=v^{\prime}$ and $q\left(k\left(k^{\prime}\right)^{-1}\right) \in Z_{q g}$. We thus have $k\left(k^{\prime}\right)^{-1} g \exp v=g \exp v k\left(k^{\prime}\right)^{-1}$. Consider the map $\alpha: V \rightarrow G$ given by $\alpha(w)=\left(g \exp w, k\left(k^{\prime}\right)^{-1}\right)$ where $($,$) denotes the commutator. We have$ $q \alpha(w)=e$ for all $w$ so that $\alpha$ is a continuous map of the connected space $V$ into the discrete space $Z$ and is constant. Since $\alpha(v)=e$, we have $\alpha(0)=e$, that is, $k\left(k^{\prime-1}\right) \in Z_{g}$. The remainder of the proof being of a local nature, it applies to the more general situation.

\section{REFERENCES}

1. E. Hewitt and K.A. Ross, Abstract harmonic analysis, vol. II, Springer-Verlag, Berlin, 1970.

2. B.E. Johnson, Cohomology in Banach algebras, Mem. Amer. Math. Soc. 127 (1972).

3. _. Derivations from $L^{1}(G)$ into $L^{1}(G)$ and $L^{\infty}(G)$, Lecture Notes in Math., vol. 1359, Springer, Berlin and New York, 1988, pp. 191-198.

Department of Mathematics and Statistics, The University, Newcastle upon Tyne, NE 17RU ENGLAND 